\title{
Methylene blue treatment for cytokine release syndrome-associated vasoplegia following a renal transplant with rATG infusion: A case report and literature review
}

\author{
JOHN T. DENNY ${ }^{1}$, ANDREW T. BURR ${ }^{1}$, FRED BALZER $^{2}$, JAMES T. TSE ${ }^{1}$, \\ JULIA E. DENNY ${ }^{3}$ and DARRICK CHYU ${ }^{1}$ \\ ${ }^{1}$ Department of Anesthesia, Robert Wood Johnson Medical School, Rutgers University, New Brunswick, NJ 08901; \\ ${ }^{2}$ Virtua Memorial Hospital, Department of Anesthesia, Mount Holly, NJ 08060; ${ }^{3}$ Department of Anesthesia, \\ New York University Medical Center, New York, NY 10016, USA
}

Received May 28, 2014; Accepted March 3, 2015

DOI: $10.3892 / \mathrm{etm} .2015 .2349$

\begin{abstract}
Rabbit anti-thymocyte globulin (rATG) is an infusion of polyclonal rabbit-derived antibodies against human thymocyte markers, which can be used to prevent and treat acute rejection following organ transplantation. However, the product monograph issued by the manufacturer (Sanofi Canada) reports that serious immune-mediated reactions have been observed following the use of rATG, consisting of anaphylaxis or severe cytokine release syndrome (CRS), which is a form of vasoplegic syndrome (VS), in which distributive shock occurs refractory to norepinephrine (NE) and vasopressin (VP). Severe infusion-associated reactions are consistent with CRS and can cause serious cardiac or respiratory problems, or in certain cases, mortality. CRS is a form of systemic inflammatory response syndrome (SIRS). In SIRS, the substantial activation of endothelial inducible nitric oxide synthase (iNOS) and smooth muscle guanylate cyclase (GC) is observed, which can produce severe hypotension that is unresponsive to conventional vasopressors. Methylene blue (MB) is a direct inhibitor of iNOS and GC and has been used as an effective treatment for VS following cardiothoracic surgery. In the present study, the successful use of MB as a rescue therapy for CRS in a patient receiving rATG following a renal transplant was reported. Following an uneventful cadaveric kidney transplant involving the intravenous (IV) administration of rATG for the induction of immunological
\end{abstract}

Correspondence to: Dr John T. Denny, Department of Anesthesia, Robert Wood Johnson Medical School, Rutgers University, Suite 3,100 Clinical Academic Building, 125 Paterson Street, New Brunswick, NJ 08901, USA

E-mail: dennyjt@rwjms.rutgers.edu

Key words: vasoplegia, vasoplegic syndrome, vasoplegic shock, distributive shock, shock, cytokines, cytokine release syndrome, thymoglobulin, renal transplant complications, rabbit anti-thymocyte globulin tolerance, the patient became markedly hypotensive and tachycardic. The patient required high doses of VP and NE infusions. Following the protocol described for treating refractory VS in post-cardiac surgery patients, the decision was made to initiate the patient on an IV MB infusion. This treatment protocol was shown to improve the hemodynamic status of the patient, which enabled the withdrawal of vasopressors and suggests an important role for methylene blue in the management of refractory VS.

\section{Introduction}

Rabbit anti-thymocyte globulin (rATG) is an infusion of polyclonal rabbit-derived antibodies against various human thymocyte markers, such as CD2, CD3, CD4, CD8, CD118, CD44, CD45, HLA-DR, HLA Class I heavy chains and $\beta_{2}$-microglobulin. The antibody infusion has been used for the prevention and treatment of acute rejection following organ transplantation; however, serious immune-mediated reactions have been reported with the use of rATG, consisting of anaphylaxis or severe cytokine release syndrome CRS $(1,2)$. Severe infusion-associated reactions (IARs) are consistent with CRS and may result in serious cardiac or respiratory compromise or mortality. CRS is a form of systemic inflammatory response syndrome (SIRS), which produces severe hypotension that is unresponsive to conventional vasopressors (4).

Vasoplegic syndrome (VS) is frequently observed in patients following a cardiopulmonary bypass. The condition is hypothesized to result from the depletion of endogenous vasopressin (VP) and the release of multiple neurohumoral and inflammatory mediators associated with SIRS, which subsequently leads to the activation of endothelial inducible nitric oxide synthase (iNOS) and smooth muscle guanylate cyclase (GC). Methylene blue (MB) is a heterocyclic aromatic compound and a direct inhibitor of NOS and GC (5).

MB is well recognized as a treatment for methemoglobinemia, and has also been described in recent years as an effective treatment for VS following cardiothoracic surgery. In patients with VS who fail to respond to norepinephrine (NE) and VP, MB is an additional therapeutic option to consider. 
The case in the present study illustrates the potential value of $\mathrm{MB}$ in the treatment of CRS.

The present study reports the successful use of $\mathrm{MB}$ as a rescue therapy for CRS, a form of VS, in which distributive shock occurred refractory to NE and VP. The patient received rATG therapy following renal transplant surgery.

\section{Case report}

The patient was a 69-year-old man with a history of hypertension, coronary artery disease, peripheral vascular disease and end-stage renal disease, receiving peritoneal dialysis. Following an uneventful cadaveric kidney transplant, which involved the intravenous (IV) administration of rATG (Thymoglobulin ${ }^{\circledR}$; Sanofi Canafa, Laval, QC, Canada) for the induction of immunological tolerance, the 69-year-old male patient became markedly hypotensive [systolic blood pressure (SBP) of $<80 \mathrm{mmHg}$ ) and tachycardic (heart rate, $130 \mathrm{bpm}$ ), although there was no evidence of sepsis or acute blood loss. The patient had a history of hypertension, coronary artery disease, peripheral vascular disease and end stage renal disease, and was receiving peritoneal dialysis. Written informed consent was obtained from the patient.

After the failure of conservative measures, including fluid challenges and the administration of phenylephrine, the patient received infusions of $\mathrm{NE}$ and arginine vasopressin (AVP), with the aim to maintain the SBP at $100 \mathrm{mmHg}$. Within $2 \mathrm{~h}$, the patient had developed hyperpyrexia with a temperature of $104^{\circ} \mathrm{F}$. Following a pan-culture, the patient was administered the empiric antibiotics, vancomycin and piperacillin-tazobactam. An infectious disease specialist reported 'no obvious infected focus on examination, nor evidence of donor infection'. By postoperative day (POD) 1, the patient continued to be hypotensive, despite a central venous pressure of $17 \mathrm{mmHg}$; thus, a pulmonary artery catheter (Swan-Ganz) was inserted (\#131HF7; Edwards Lifesciences Corporation, Irvine, CA, USA). Initial measurements yielded a pulmonary capillary wedge pressure of $18 \mathrm{mmHg}$, a cardiac index of $4.0 \mathrm{l} / \mathrm{min} / \mathrm{m}^{2}$ and a systemic vascular resistance (SVR) of 491 dynes $\cdot \mathrm{sec}^{\cdot} \mathrm{cm}^{-5}$, while receiving a high dose of AVP and $\mathrm{NE}(0.09 \mathrm{U} / \mathrm{min}$ and $0.17 \mu \mathrm{g} / \mathrm{kg} / \mathrm{min}$, respectively).

A non-ST segment elevation myocardial infarction was suspected, with a peak troponin level of $12 \mathrm{ng} / \mathrm{ml}$. However, a bedside transthoracic echocardiogram revealed an ejection fraction of $60 \%$, with normal left ventricular wall motion and contractility.

Later on POD 1, the patient continued to require high doses of AVP $(0.09 \mathrm{IU} / \mathrm{min})$ and $\mathrm{NE}(0.17 \mu \mathrm{g} / \mathrm{kg} / \mathrm{min})$ in order to maintain an SBP of $>80 \mathrm{mmHg}$. Thus, following the protocol described for treating refractory VS in post-cardiac surgery patients, the decision was made to initiate the patient on an IV infusion of $\mathrm{MB}$.

Prior to the initiation of MB therapy, the NE infusion was set at $0.17 \mu \mathrm{g} / \mathrm{kg} / \mathrm{min}$ and the AVP infusion was set at $0.09 \mathrm{IU} / \mathrm{min}$. Following a partial load of $\mathrm{MB}(0.5 \mathrm{mg} / \mathrm{kg})$, the hemodynamic status of the patient improved sufficiently and the AVP was immediately reduced by $56 \%$ to $0.04 \mathrm{IU} / \mathrm{min}$. Following application of the remainder of the loading dose (total, $1.5 \mathrm{mg} / \mathrm{kg}$ ), the NE dose was decreased by $41 \%$ to $0.10 \mu \mathrm{g} / \mathrm{kg} / \mathrm{min}$. By POD 2, the NE infusion was withdrawn altogether, and the MB was titrated off. In addition, the AVP infusion was withdrawn by POD 3. All cultures remained negative. The patient was transferred from the intensive case unit to the hospital ward on POD 7. After 7 months the patient succumbed to sepsis and multiple system organ failure in another facility.

\section{Discussion}

rATG or Thymoglobulin ${ }^{\circledR}$ is an infusion of polyclonal rabbit-derived antibodies targeted against human thymocyte markers, which has been used for the prevention and treatment of acute rejection following organ transplantation (3). However, serious immune-mediated reactions have been reported with the use of rATG, including anaphylaxis or severe CRS. Fatal cases of anaphylaxis have also been reported. Severe IARs are consistent with CRS and can cause serious cardiac or respiratory compromise, or in certain cases, mortality (1). CRS is a form of SIRS, and as aforementioned, SIRS can result in severe hypotension that is unresponsive to treatment with conventional vasopressors.

Vasodilatory shock that is refractory to high-dose NE has been described in the literature as VS. The condition is frequently observed in patients following a cardiopulmonary bypass (4), and is hypothesized to result from the depletion of endogenous VP (5) and the release of multiple neurohumoral and inflammatory mediators associated with SIRS (4-10), which subsequently leads to the activation of endothelial iNOS and smooth muscle GC. MB is a heterocyclic aromatic compound that is well known as a treatment for methemoglobinemia, and has also been described in recent years as an effective treatment for vasopressor-resistant vasoplegia following cardiothoracic surgery $(8,9)$.

The pharmacological mechanism underlying the effects of $\mathrm{MB}$ in the context of SIRS-associated VS is that MB exerts its effect at the level of the 'final common pathway' in the distributive shock cascade. Therefore, theoretically, the benefit of $\mathrm{MB}$ is realized independent of the etiology of the upstream inflammatory insult $(9,10)$.

Inflammatory mediators in SIRS activate iNOS, increasing the production of nitric oxide (NO). Subsequently, the soluble GC of smooth muscle cells is activated and cyclic GMP (cGMP) production is increased. The increased levels of NO and cGMP lead to profound vasodilatation, among a host of additional effects (10).

$\mathrm{MB}$ is a direct inhibitor of iNOS and GC, and has been shown to be an effective treatment for VS following cardiothoracic surgery. In addition, MB has been demonstrated to be effective for patients with distributive shock caused by sepsis or anaphylaxis that is refractory to NE and VP (10-12).

A number of anecdotal studies have described the use of $\mathrm{MB}$ in reversing vasoplegia $(4,6,7)$. However, there have been a limited number of randomized, controlled studies investigating the use of MB in patients with vasodilation. Flynn and Sladen discussed three studies that identified significant increases in the mean arterial pressure and no adverse effects following the administration of MB (11). The largest trial was performed by Levin et al, which included post-cardiac surgery patients with postoperative VS. The authors found that the use of MB not only reduced the duration of vasoplegia to $<6 \mathrm{~h}$ in 
all the patients, but also reduced the high mortality rate in the sample population (12).

Several clinical trials using MB have been performed, and all the trials have demonstrated that MB increases the SVR, as reflected by an increase in the mean arterial pressure or by a decrease in vasopressor requirement $(5,9,11,12,14)$. Notably, a number of the trials demonstrated increased pulmonary vascular resistance with $\mathrm{MB}$; however, these trials used large bolus doses, not the recommended infusions at lower doses. Inhaled NO has been proposed to counteract any potential increases in pulmonary vascular resistance that are associated with MB. The selectivity of MB for iNOS indicates the benefits and predisposition of this therapeutic option (11).

Despite the lack of a uniform definition, the terms 'vasoplegia' and 'vasoplegic syndrome (VS)' are being used increasingly in the literature to describe a state of profound vasodilatory shock (5). This condition is characterized by a markedly decreased SVR and vasomotor hyporeactivity that persists despite a high cardiac output, ample volume resuscitation and high-dose NE administration $(6,7)$.

Such catecholamine-resistant distributive shock states, which are manifestations of severe SIRS (6), have been described in diverse clinical contexts in response to a variety of etiological triggers (7). However, at a molecular level, the different forms appear to share the same final common pathway, namely unregulated NO synthesis and the resultant overproduction of cGMP in vascular smooth muscle (4-7).

Numerous factors have been implicated as contributing to circulatory failure in SIRS, including endothelial injury, the depletion of endogenous AVP $(5,7)$ and the release of multiple neurohumoral and inflammatory mediators $(4-7,10)$, such as prostaglandin I2 $(5,7)$, bradykinin $(5,7)$, interleukin (IL)-1 $\beta$ (13) and atrial natriuretic peptide (ANP) (7), which directly activate smooth muscle GC (5-7,10,14-16).

In the case of refractory vasoplegia, high levels of circulating IL-1 $\beta$, IL-6, tumor necrosis factor (TNF)- $\alpha$, interferon- $\gamma$, platelet-activating factor and adenosine result in the substantial induction of endothelial iNOS, leading to unregulated NO synthesis $(4,5,17-20)$ and widespread activation of GC in vascular smooth muscle. The resulting high levels of NO and cGMP within the vascular myocytes causes changes at the subcellular level, which directly give rise to the vasoplegic state. In this state, $\mathrm{K}^{+}$channels are opened for membrane hyperpolarization, while voltage-gated $\mathrm{Ca}^{2+}$ channels are closed, resulting in the dephosphorylation of myosin, and subsequently, myocyte relaxation (5). Furthermore, angiotensin II and NE enable vasoconstriction by increasing the calcium entry into the vascular myocytes; NO-mediated myocyte hyperpolarization and $\mathrm{Ca}^{2+}$ channel closure inhibit the very mechanism through which these vasopressors exert their effect. Therefore, hypotension and vasodilation persist, despite the high plasma concentrations of these hormones (5). A concomitant increase in cGMP levels in cardiac myocytes leads to a decrease in myocardial contractility $(17,21,22)$.

ANP activates membrane bound GC, while NO, IL-1 $\beta$ and carbon monoxide (CO) activate soluble GC. In addition, $\mathrm{CO}$ activates iNOS directly, and is produced within the cells from the breakdown of heme by heme oxygenase $(17,22)$.

The term 'vasoplegic syndrome (VS)' was coined to describe this phenomenon. To date, VS has received substantial attention in cardiac surgery literature, where the condition is reported to occur in up to $10 \%$ of patients following a cardiopulmonary bypass, and in up to $42 \%$ of patients following a left ventricular assist device placement for end stage heart failure $(4,8,9,12,23)$. However, VS is being increasingly recognized in other clinical contexts, such as in association with anaphylaxis (including anaphylactoid protamine reactions) (20,24-27), septic shock $(5,17,28)$, decompensated hemorrhagic shock $(5,7,29)$, hemodialysis (30), heart and liver transplantation $(10,22)$ and CRS associated with the IV administration of rATG (Thymoglobulin ${ }^{\circledR}$ ), monoclonal anti-CD3 or anti-CD20 (31-33). Furthermore, in recent years, VS has been recognized as the final common pathway for long-lasting severe shock of any cause (5).

Risk factors for VS following cardiac surgery include the preoperative administration of angiotensin-converting-enzyme inhibitors, calcium channel blockers, amiodarone or IV heparin, as well as conditions marked by high levels of endogenous catecholamines, such as congestive heart failure and acute myocardial infarction, and/or endothelial dysfunction, such as diabetes mellitus $(4,5,7,17,18)$.

VS is associated with a poor prognosis $(7,10)$. In studies of patients with VS following a cardiac pulmonary bypass, the duration of vasodilatory shock was shown to significantly influence the overall morbidity and mortality rates. Mortality rates as high as $25 \%$ were reported in cardiac surgery patients when postoperative vasoplegia persisted for $>36 \mathrm{~h}(13,19)$. Furthermore, in patients with septic shock, VS is present in $\sim 50 \%$ of the patients who succumb to sepsis, and the condition is the leading cause of mortality in the first week following diagnosis $(4,7,15,16)$.

Faced with these poor statistics, it is worth considering two statements made previously: i) 'In recent years, VS has been recognized as the final common pathway for long-lasting severe shock of any cause (5)'; and ii) 'At the molecular level, VS is caused by a massive induction of endothelial iNOS, leading to unregulated NO synthesis and widespread activation of GC in vascular smooth muscle (4-7)'. Taken together, these statements indicate the possibility that a treatment demonstrated to reverse VS in one clinical context, by safely antagonizing NO and GC, may be equally effective in reversing vasoplegia in any other clinical scenario.

One such treatment is AVP. AVP, or antidiuretic hormone, is a small peptide that is released from the posterior pituitary in response to a reduction in effective circulating volume, arterial hypotension or an increase in serum osmolality. AVP is an extremely potent vasoconstrictor. However, under normal physiological conditions, any increase in circulating AVP functions centrally to bias the arterial baroreflex in the sympathoinhibitory direction, subsequently buffering its own pronounced vasoconstrictive effects (21).

Serum osmolality is the main feedback signal to the hypothalamus, which modulates AVP release. However, during conditions that reduce the cardiac preload, a hypovolemic stimulus can override osmotic control, with the result that AVP is released despite marked hyponatremia (hypo-osmolality). Very high plasma concentrations of endogenous AVP can be attained during volume depletion and hypotension (20-400 pg/ml) (21,34). As previously discussed, endogenous AVP becomes depleted as the shock state persists $(5,34)$, which 
provides a rationale for the exogenous administration of VP in patients with advanced vasodilatory shock $(8,35)$.

In vascular smooth muscle, AVP counteracts the effects of NO and cGMP via the G-protein coupled vasopressin $1 \mathrm{~A}$ receptor. Binding of AVP to the vasopressin 1A receptoractivates the phospholipase $\mathrm{C}$ /inositol triphosphate (increasing intracellular $\mathrm{Ca}^{2+}$ ) and protein kinase $\mathrm{C} /$ myosin light-chain kinase (increasing myosin phosphorylation) signaling cascades, which partially restores vascular smooth muscle contractility, and partially reverses the NO- and cGMP-mediated resistance to $\mathrm{NE}$ and angiotensin II.

Since the 1990s, AVP has been used as an adjunct vasopressor in patients with severe vasodilatory shock or vasoplegia. While AVP has been repeatedly demonstrated to be effective at improving the hemodynamic status in catecholamine-resistant shock states, no survival benefit has been observed at doses between 0.01 and $0.03 \mathrm{IU} / \mathrm{min}$, as reported by the Vasopressin in Septic Shock Trial (VASST) (35). However, at these doses, subgroup analysis revealed that AVP improved the outcome in patients with less severe septic shock and in patients receiving low-dose corticosteroids.

The Surviving Sepsis Campaign guidelines indicate that AVP may be added as a supplementary vasopressor when hemodynamic instability persists, despite escalating NE doses (36).

Although multiple studies have evaluated the therapeutic effects of AVP in vasodilatory shock (34-36), an optimal dose has yet to be established. The authors of the VASST study selected AVP infusion rates of 0.01-0.03 IU/min (35), while the Surviving Sepsis Campaign guidelines recommend infusing AVP at $0.03 \mathrm{IU} / \mathrm{min}$ (36). More recently, however, an open label randomized controlled trial comparing IV AVP at two dose rates $(0.033$ and $0.067 \mathrm{IU} / \mathrm{min})$ in patients with vasoplegia, demonstrated that while both infusion rates led to an increased mean arterial pressure $(>69 \pm 14 \mathrm{mmHg})$ and decreased heart rate, the higher dose rate $(0.067 \mathrm{IU} / \mathrm{min})$ led to a more pronounced reduction in NE requirement, with no significant increase in adverse events, as compared with the low-dose (0.033 IU/min) group (35). This result indicates that AVP dose rates that are much higher than the currently recommended level may be effective at reversing cardiovascular failure in patients with advanced vasodilatory shock (35). In addition, coadministration of IV glucocorticoids appears to significantly potentiate the beneficial effects of AVP (35).

An additional approach that has been attempted is the direct inhibition of NOS with nonselective inhibitors. Tilarginine [L-(N)-monomethyl arginine or NG-monomethyl-L-arginine $\mathrm{HCl}]$ is a nonselective inhibitor of NOS that has been studied in the treatment of vasoplegia-complicated sepsis and post-myocardial infarction cardiogenic shock (37). Despite clear evidence that the overproduction of NO plays a pivotal role in the pathogenesis of vasoplegia-associated septic shock and myocardial infarction complicated with refractory cardiogenic shock, outcome studies in these two disorders have produced disappointing results $(27,28)$.

A phase III trial investigating the effect of tilarginine on patients with septic shock was terminated early due to the increased mortality rate in the treatment group, as compared with the placebo group. At day 28 , the mortality rate was $59 \%$ in the tilarginine treatment group, as compared with
$49 \%$ in the patients receiving the placebo $(\mathrm{P}<0.005)$. Overall, the adverse effects were similar between the placebo and treatment groups; however, the patients receiving tilarginine experienced a higher incidence of cardiovascular events, including pulmonary hypertension, systemic hypertension, decreased cardiac output and heart failure. Causes of mortality were similar to those expected in patients with severe sepsis; however, a higher percentage of fatalities referable to cardiovascular events were associated with the tilarginine treatment (37). To aid the interpretation of these results, it is worth reviewing the various roles of NOS in the cardiovascular system.

NO is produced by three isoforms of NOS, namely neuronal (nNOS), endothelial (eNOS) and iNOS. These three isoforms are products of different genes and have different localizations, kinetics, regulation properties and roles in neuronal, immune, vascular and myocardial physiology. The first two isoforms are constitutively expressed, fast-acting enzymes, whose NO production is calcium-dependent. The third isoform, iNOS, is a slow-acting, high-output, calcium-independent enzyme. Activated iNOS generates large amounts of NO by a sustained production for up to $10 \mathrm{~h}$. All three isoforms of NOS are expressed in the myocardium, where eNOS and nNOS contribute to sustaining normal excitation-contraction coupling and contribute to the Frank-Starling mechanism. The enzymes also moderate the $\beta 1-/ \beta 2$-adrenergic increase in inotropy and chronotropy, and reinforce the vagal control of cardiac contraction, thereby protecting the heart against excessive stimulation by catecholamines, such as endogenous $\beta$-blockers (16-18,22,24,25).

Non-isoform-specific inhibition of NO production in the presence of excessive amounts of endogenous and exogenous vasoconstrictor agents potentiates the vasoconstrictor effects of catecholamines and may result in excessive vasoconstriction, compromising myocardial and systemic tissue perfusion. Numerous experimental studies have demonstrated peripheral and coronary vasoconstriction, reduced cardiac output and myocardial dysfunction via nonselective NOS inhibition. In experimental models of acute myocardial infarction, nonselective NOS inhibition with N-nitro-L-arginine was associated with the exaggeration of myocardial dysfunction due to a reduction in regional blood flow in the surviving myocardium. In addition, elimination of endogenous NO synthesis has been shown to exacerbate myocardial stunning and reduce the percentage of perfused capillaries in a normal and stunned myocardium. Patients with cardiogenic shock may be particularly sensitive to slight reductions in the coronary blood flow to the surviving myocardium. Furthermore, the elevation in blood pressure that follows NOS inhibition may be the result of increased systemic vascular resistance that reduces cardiac output $(16,17,22,24,25)$.

Previous results demonstrate that treatment of sepsis with nonselective inhibitors of NO originating from L-arginine are associated with unfavorable cardiac effects and a higher mortality rate (28). Due to these findings, further research studies with these compounds are unlikely to be initiated. However, promising results from in vitro and animal studies have shown that continued research into the function of these compounds is warranted $(38,39)$. Initial studies of NO scavengers revealed encouraging hemodynamic effects, minus the 
evident adverse cardiac consequences; thus, further investigation is required.

Diaspirin cross-linked hemoglobin (DCLHb) is a stroma-free hemoglobin solution that has been shown to cause reproducible vasoconstriction at low doses in animal and healthy human subjects (40). The mechanism underlying the vasopressor effect is yet to be fully elucidated; however, it is hypothesized that DCLHb accumulates NO, in addition to changing the sensitivity of the adrenergic receptor (40). A pilot study of DCLHb was conducted in 14 patients with a low SVR due to sepsis syndrome who required vasopressor treatment to maintain their blood pressure. DCLHb was administered in up to five $100-\mathrm{ml}$ doses to a maximum dose of $700 \mathrm{mg} / \mathrm{kg}$. A rapid and profound vasopressor response was observed in all the test subjects. As a result, doses of conventional vasopressor usage were decreased by an average of $50 \%(15-100 \%)$. With regard to the laboratory evaluations, base deficit and lactic acid levels did not change, no deleterious findings were observed and the cardiac output was unchanged (40).

$\mathrm{MB}$ is a chemical dye that can compete with NO by crossing the cell membrane and subsequently inhibiting the activation of soluble GC by binding with the iron heme moiety in GC. Despite numerous case reports investigating MB for the reversal of VS, a limited number of randomized, controlled studies using MB in vasoplegic patients have been conducted. To date, three studies have demonstrated a significant improvement in the mean arterial pressure, without any observed adverse effects $(7,8,12)$. Levin et al investigated MB treatment in post-cardiac surgery patients with VS. MB administration was shown to decrease the length of vasoplegia to $<6 \mathrm{~h}$ in all the patients and also reduce the mortality rate in the sample population (12).

Clinical trials with MB have demonstrated that the drug increases the SVR, manifesting as an increase in blood pressure or as a decrease in the vasopressor dosage. A small number of trials have demonstrated an elevation in pulmonary vascular resistance with MB; however, these trials used bigger bolus dosages, rather than the recommended IV administration at a lower rate $(5,41)$. Inhaled NO is hypothesized to counteract any possible increases in the pulmonary vascular resistance that may occur following MD administration. However, the affinity of MD for iNOS may make the drug a preferred choice of therapy.

In contrast to nonselective NOS inhibitors, MB has been shown to exert beneficial effects in vasoplegic patients. MB may also produce positive results in vasoplegic patients via the inhibitory effects of molecules, such as TNF- $\alpha$, which lead to an improved myocardial output. Furthermore, MB has been demonstrated to have salutatory effects in inhibiting superoxide radical formation through competing with oxygen for electron transfer via xanthine oxidase (11). In summary, MB has been demonstrated to exert a positive effect in reversing vasodilatory shock.

$\mathrm{MB}$ inhibits the effects of NO in vascular tissues, while not altering the quantity of $\mathrm{NO}$ produced. The effects of $\mathrm{MB}$ on NO were reviewed in four randomized, clinical trials with small patient populations. MB was administered in bolus doses of $1-3 \mathrm{mg} / \mathrm{kg}$ over $20 \mathrm{~min}$, while the blood pressure and cardiac parameters were assessed for the subsequent $3 \mathrm{~h}$. All four studies revealed significant increases in the SVR and mean arterial pressure $(12,17,21,41)$. In addition, three studies demonstrated no change in oxygen delivery, oxygen consumption and the cardiac index, while one study observed increases in these parameters $(12,17,21)$. The study that used the highest dose of $\mathrm{MB}(3 \mathrm{mg} / \mathrm{kg})$ observed a decrease in the pulmonary artery pressures, pulmonary vascular resistance and $\mathrm{PaO}_{2}: \mathrm{FiO}_{2}$ ratios. Furthermore, one group observed a decrease in lactate measurements; however, the authors concluded that this was a result of the $\mathrm{MB}$, instead of an improvement in cellular metabolism (41). A continuous infusion of MB was determined to be necessary, since the positive effects of the MB bolus were observed to last for only $3 \mathrm{~h}$ (12).

$\mathrm{MB}$ is a heterocyclic aromatic compound. Among its numerous clinical uses, $\mathrm{MB}$ has been demonstrated to be very effective as a treatment for patients with VS following cardiothoracic surgery, as well as for patients with VS arising in the context of liver transplantation, sepsis, anaphylaxis and hemodialysis $(5,6,17,21,38,42)$.

MB rapidly reverses VS by scavenging NO via the deactivation of the Fe-Heme prosthetic group in soluble GC. Thus, MB decreases the levels of NO and cGMP in vascular smooth muscle, which reverses vasodilation and reestablishes vasomotor responsiveness to NE and angiotensin II. An increase in blood pressure is observed following the administration of MB to a patient with septic shock, which is the result of the return of peripheral vascular tone and the improvement in cardiac contractility. In addition, MB inhibits the formation of superoxide and peroxynitrite; thus, significantly decreases the harmful effects associated with myocardial or cerebral ischemia and reperfusion $(5,6,17,21,38,42)$.

A randomized trial assessing the use of MB therapy for patients with vasoplegia following cardiac surgery demonstrated a statistically significant decrease in the mortality rate (0\% in the MB group vs. $21.4 \%$ in the control group) (17). Furthermore, the MB treatment group exhibited a significant reduction in the incidence of renal failure, respiratory failure, myopathy, neuropathy, supraventricular arrhythmia, sepsis, and multi-organ dysfunction (17).

With regard to the pharmacological mechanism underlying the effects of MB in the context of SIRS associated with VS, $\mathrm{MB}$ is hypothesized to exert its effect at the level of the 'final common pathway' in the distributive shock cascade. Therefore, theoretically, the benefit of MB may be realized independent of the etiology of the upstream inflammatory insult. The inducible isoform of NOS is activated by inflammatory mediators that are produced during SIRS, which subsequently increases the production of NO. In turn, soluble GC of the smooth muscle cells is activated, increasing the production of cGMP. Increases in the levels of NO and cGMP lead to significant vasodilatory and other effects, as discussed previously.

Therefore, MB directly inhibits NOS and GC, and has been shown to have a beneficial effect for patients with distributive shock refractory to NE and VP. In addition, MB may be a successful therapy for patients with VS following cardiac surgery, sepsis or anaphylaxis.

In conclusion, $\mathrm{MB}$ is a potential therapeutic option to consider in patients with vasoplegic shock who fail to respond to catecholamine and VP therapy. However, further research is required to determine whether certain patients may benefit 
from treatment with MB more, and to assess when the side effects may outweigh the benefits.

\section{Acknowledgements}

Sections of this case report were presented as a poster at the Society of Critical Care Medicine 39th Annual Congress, Jan 2010: Denny J, Morgan S, Denny JT, Slomovits M and Balzer F: Methylene blue as rescue treatment in SIRS following renal transplant. Crit Care Med 37: 12S, 2009.

\section{References}

1. Teachey DT, Rheingold SR, Maude SL, et al: Cytokine release syndrome after blinatumomab treatment related to abnormal macrophage activation and ameliorated with cytokine-directed therapy. Blood 121: 5154-5157, 2013.

2. Sanofi Canada: Thymoglobulin ${ }^{\circledR}$ (Anti-thymocyte globulin [rabbit]) product monograph. http://products.sanofi.ca/en/thymoglobulin.pdf. Accessed Jan 25, 2015.

3. Mahmud N, Klipa D and Ahsan N: Antibody immunosuppressive therapy in solid-organ transplant: Part I. MAbs 2: 148-156, 2010

4. Shanmugam G: Vasoplegic syndrome - the role of methylene blue. Eur J Cardiothorac Surg 28: 705-710, 2005.

5. Landry DW and Oliver JA: The pathogenesis of vasodilatory shock. N Engl J Med 345: 588-595, 2001.

6. Friedrich M, Bräuer A, Tirilomis T, Lotfi S, Mielck F and Busch T: Methylene blue administration in severe systemic inflammatory response syndrome (SIRS) after thoracic surgery. Ann Thorac Cardiovasc Surg 8: 306-310, 2002.

7. Stawicki SP, Sims C, Sarani B, Grossman MD and Gracias VH: Methylene blue and vasoplegia: who, when, and how? Mini Rev Med Chem 8: 472-490, 2008.

8. Argenziano M, Chen JM, Cullinane S, et al: Arginine vasopressin in the management of vasodilatory hypotension after cardiac transplantation. J Heart Lung Transplant 18: 814-817, 1999.

9. Taylor KM: SIRS - the systemic inflammatory response syndrome after cardiac operations. Ann Thorac Surg 61: 1607-1608, 1996.

10. Downing SW and Edmunds LH Jr: Release of vasoactive substances during cardiopulmonary bypass. Ann Thorac Surg 54: 1236-1243, 1992.

11. Flynn BC and Sladen RN: The use of methylene blue for vasodilatory shock in a pediatric lung transplant patient. J Cardiothorac Vasc Anesth 23: 529-530, 2009.

12. Levin RL, Degrange MA, Bruno GF, et al: Methylene blue reduces mortality and morbidity in vasoplegic patients after cardiac surgery. Ann Thorac Surg 77: 496-499, 2004.

13. Beasley D and McGuiggin M: Interleukin 1 activates soluble guanylate cyclase in human vascular smooth muscle cells through a novel nitric oxide-independent pathway. J Exp Med 179: 71-80, 1994.

14. Mekontso-Dessap A, Houël R, Soustelle C, Kirsch M, Thebért D and Loisance DY: Risk factors for post-cardiopulmonary bypass vasoplegia in patients with preserved left ventricular function. Ann Thorac Surg 71: 1428-1432, 2001.

15. Robin JK, Oliver JA and Landry DW: Vasopressin deficiency in the syndrome of irreversible shock. J Trauma 54 (Suppl): S149-S154, 2003.

16. Boyle EM Jr, Pohlman TH, Johnson MC and Verrier ED: Endothelial cell injury in cardiovascular surgery: the systemic inflammatory response. Ann Thorac Surg 63: 277-284, 1997.

17. Kwok ES and Howes D: Use of methylene blue in sepsis: A systematic review. J Intensive Care Med 21: 359-363, 2006.

18. Cauwels A, Janssen B, Buys E, Sips P and Brouckaert P: Anaphylactic shock depends on PI3K and eNOS-derived NO. J Clin Invest 116: 2244-2251, 2006.

19. Montrucchio G, Alloatti G and Camussi G: Role of platelet-activating factor in cardiovascular pathophysiology. Physiol Rev 80: 1669-1699, 2000.

20. Neugut AI, Ghatak AT and Miller RL: Anaphylaxis in the United States: an investigation into its epidemiology. Arch Intern Med 161: 15-21, 2001.

21. Gomes WJ, Carvalho AC, Palma JH, et al: Vasoplegic syndrome after open heart surgery. J Cardiovasc Surg (Torino) 39: 619-623, 1998.
22. Schulz R, Nava E and Moncada S: Induction and potential biological relevance of a $\mathrm{Ca}(2+)$-independent nitric oxide synthase in the myocardium. Br J Pharmacol 105: 575-580, 1992.

23. Cremer J, Martin M, Redl H, et al: Systemic inflammatory response syndrome after cardiac operations. Ann Thorac Surg 61: 1714-1720, 1996.

24. Brown SG: Cardiovascular aspects of anaphylaxis: implications for treatment and diagnosis. Curr Opin Allergy Clin Immunol 5: 359-364, 2005.

25. Kemp SF and Lockey RF: Anaphylaxis: a review of causes and mechanisms. J Allergy Clin Immunol 110: 341-348, 2002.

26. Takakura K, Mizogami M and Fukuda S: Protamine sulfate causes endothelium-independent vasorelaxation via inducible nitric oxide synthase pathway. Can J Anaesth 53: 162-167, 2006.

27. Viaro F, Dalio MB and Evora PR: Catastrophic cardiovascular adverse reactions to protamine are nitric oxide/cyclic guanosine monophosphate dependent and endothelium mediated: should methylene blue be the treatment of choice? Chest 122: 1061-1066, 2002.

28. Donati A, Conti G, Loggi S, et al: Does methylene blue administration to septic shock patients affect vascular permeability and blood volume? Crit Care Med 30: 2271-2277, 2002.

29. Thiemermann C, Szabó C, Mitchell JA and Vane JR: Vascular hyporeactivity to vasoconstrictor agents and hemodynamic decompensation in hemorrhagic shock is mediated by nitric oxide. Proc Natl Acad Sci USA 90: 267-271, 1993.

30. Peer G, Itzhakov E, Wollman Y, et al: Methylene blue, a nitric oxide inhibitor, prevents haemodialysis hypotension. Nephrol Dial Transplant 16: 1436-1441, 2001.

31. Guttmann RD, Caudrelier P, Alberici G and Touraine JL: Pharmacokinetics, foreign protein immune response, cytokine release, and lymphocyte subsets in patients receiving thymoglobuline and immunosuppression. Transplant Proc 29: 24S-26S, 1997.

32. Jeyarajah DR and Thistlethwaite JR, Jr.: General aspects of cytokine-release syndrome: timing and incidence of symptoms. Transplant Proc 25 (Suppl 1): 16-20, 1993.

33. Radhakrishnan J and Cohen DJ: Cytokine-release syndrome: general risk-factor modification - preparation of high-risk patients for use of OKT3. Transplant Proc 25 (Suppl 1): 60-62, 1993.

34. Rosenzweig EB, Starc TJ, Chen JM, et al: Intravenous arginine-vasopressin in children with vasodilatory shock after cardiac surgery. Circulation 100 (Suppl): II182-II186, 1999.

35. Torgersen C, Dünser MW, Wenzel V, et al: Comparing two different arginine vasopressin doses in advanced vasodilatory shock: a randomized, controlled, open-label trial. Intensive Care Med 36: 57-65, 2010.

36. Dellinger RP, Levy MM, Rhodes A, et al; Surviving Sepsis Campaign Guidelines Committee including the Pediatric Subgroup: Surviving sepsis campaign: international guidelines for management of severe sepsis and septic shock: 2012. Critical Care Med 41: 580-637, 2013

37. Alexander JH, Reynolds HR, Stebbins AL, et al; TRIUMPH Investigators: Effect of tilarginine acetate in patients with acute myocardial infarction and cardiogenic shock: the TRIUMPH randomized controlled trial. JAMA 297: 1657-1666, 2007.

38. Grubb KJ, Kennedy JL, Bergin JD, Groves DS and Kern JA: The role of methylene blue in serotonin syndrome following cardiac transplantation: a case report and review of the literature. J Thorac Cardiovasc Surg 144: e113-e116, 2012.

39. Licker M, Diaper J, Cartier V, et al: Clinical review: management of weaning from cardiopulmonary bypass after cardiac surgery. Ann Card Anaesth 15: 206-223, 2012.

40. Sloan EP, Koenigsberg MD, Philbin NB and Gao W; DCLHb Traumatic Hemorrhagic Shock Study Group; European HOST Investigators: Diaspirin cross-linked hemoglobin infusion did not influence base deficit and lactic acid levels in two clinical trials of traumatic hemorrhagic shock patient resuscitation. J Trauma 68: 1158-1171, 2010.

41. Leyh RG, Kofidis T, Strüber M, et al: Methylene blue: the drug of choice for catecholamine-refractory vasoplegia after cardiopulmonary bypass? J Thorac Cardiovasc Surg 125: 1426-1431, 2003.

42. Fischer GW and Levin MA: Vasoplegia during cardiac surgery: current concepts and management. Semin Thorac Cardiovasc Surg 22: 140-144, 2010. 